\title{
A stochastic version of the Pedersen-Sherman insulin secretion model
}

\author{
Ankit Dwivedi
}

Project Advisor: Dr. Pranay Goel

\begin{abstract}
Pedersen and Sherman have recently developed a multi-pool model of insulin vesicle secretion from pancreatic beta-cells [12]. In the PedersenSherman model, pool sizes are reported as concentrations; however, concentrations of the different pools vary by as much as seven orders of magnitude. Very low concentrations indicate there could be discrete numbers of vesicles in some of the pools, leading naturally to the questions regarding stochasticity in the signalling pathway. We therefore simulate a discrete counterpart of the deterministic model with a (hybrid) Gillespie-style stochastic simulation algorithm. The stochastic simulations are calibrated to the deterministic model in the mean. We estimate the variances in pool sizes numerically and show it closely tracks the mean, indicating a Poisson-like result. Our numerical results demonstrate that the mean behavior indicated in the Pedersen-Sherman model is evident with just one islet.
\end{abstract}

Keywords Insulin secretion - Stochastic · Deterministic · Gillepie SSA • Variance $\cdot$ Mean

\section{Introduction}

Insulin is a hormone of the body that underlies energy regulation. The betacell in the pancreatic islets of Langerhans is the site where insulin is produced and packaged for release into the blood by a process called exocytosis. Insulin secretion is mediated by calcium influx into the cell which raises cytosolic concentrations. Insulin is folded into secretory "vesicles" deep in the cell in an

A. Dwivedi

Indian Institute of Science Education and Research, Pune, Maharashtra-411021, India

E-mail: ankit.dwivedi@alumni.iiserpune.ac.in

P. Goel

Indian Institute of Science Education and Research, Pune, Maharashtra 411021, India

E-mail: pgoel@iiserpune.ac.in 
organelle called the Golgi apparatus, and is transported to the cell membrane to be readied for secretion. Insulin vesicles pass through a sequence of stages of maturation in order to complete the process of binding with the membrane before final release, as shown in Fig. 1. These "pools" typically contain a few vesicles each. The kinetics of movement of vesicles between the pools is not fully understood, and mathematical modeling is one approach used to complement experimentation in this direction.

It is common practice in biochemistry to write down differential equations for such reactions, assuming mass action kinetics is an effective model. These equations are thus rooted in a continuum assumption, that the pools are well represented as concentrations. However, such an assumption needs to be reexamined when "copy numbers" of a specie (such as the insulin vesicles in the various pools) are too few in number to warrant a concentration viewpoint. The typical method of replacing the deterministic ordinary differential equations (ODEs) is the use of stochastic simulations due to Gillespie, called the stochastic simulation algorithm (SSA). The SSA tracks the progress of the particles of a specie in a manner statistically consistent with the reaction rate equations. In the common applications of the SSA, however, reaction rates are typically constant, i.e. independent of time.

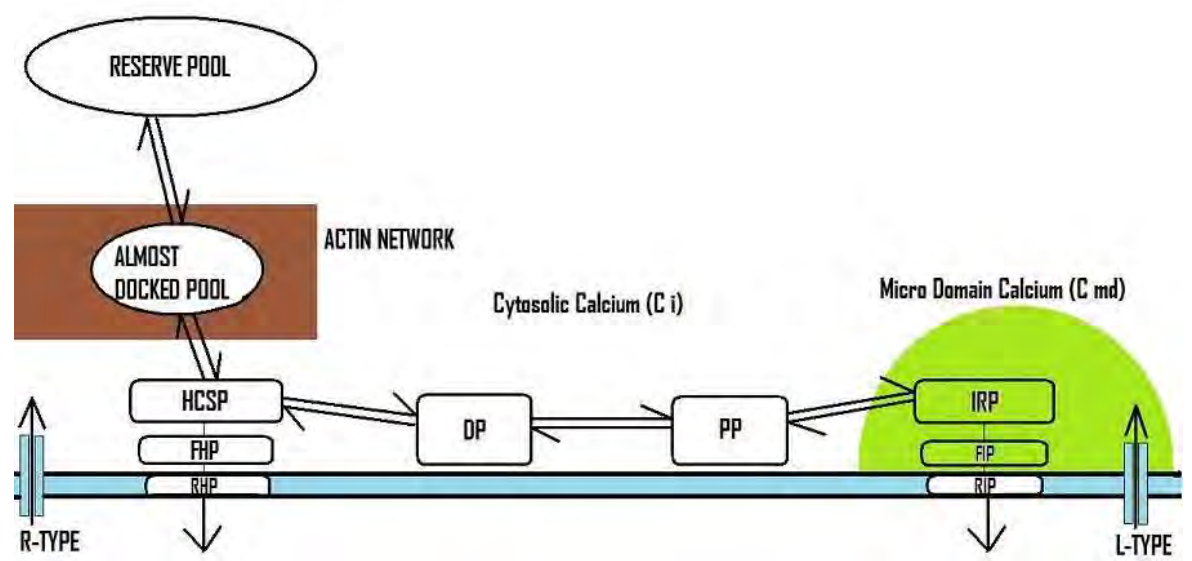

Fig. 1 Schematic overview of the model. As explained by Pedersen and Sherman in [12], the granules from reserve pool (RP) move towards the membrane passing through almostdocked Pool (AP). Before docking to the membrane vesicles tether weakly with high affinity for cytosolic calcium and are said to be in the highly calcium sensitive pool (HCSP). Then, maturing further vesicles go through docking and priming and are said to be in docked pool (DP) and primed pool (PP), respectively. After priming the vesicles are in very close vicinity of the calcium channels having low affinity for microdomain calcium and are said to be in the immediately releasable pool(IRP). FHP and FIP are the fusion pools of HCSP and IRP, respectively. RHP and RIP are the releasing pools of HCSP and IRP , respectively.

In a cell, vesicles are held in a large reserve pool (RP) from which they move towards the membrane through an almost docked pool (AP). Here granules are 
tethered weakly to the membrane with a very high affinity for cytosolic calcium $\left(C_{i}\right)$ and are said to be in the highly calcium sensitive pool (HCSP). Maturing further the granules undergo docking (DP) and then priming (PP). The primed granules progress further into an immediately-releasable pool (IRP) in which the granules are situated close to L-type calcium channels, and fuse with a low affinity to microdomain calcium $\left(C_{m d}\right)$. FHP and FIP are fusion pools of HCSP and IRP, respectively. Once granules are fused the fusion pore expands and the vesicles enter into releasing pools, RHP and RIP of HCSP and IRP, respectively. The docked pool, primed pool and the immediately releasable pool are collectively identified as the readily releasable pool (RRP) [12].

In [12], the authors have constructed a mathematical model of insulin vesicle maturation and secretion in cells in islets of Langerhans. The reaction overview of the multi-pool model of insulin vesicle secretion is as shown in Fig. 2. The model is modified from earlier work $[2,12]$, and includes nine consecutive vesicle pools representing granules in different states. The dynamics of each vesicle pool is represented by ODEs and is recapitulated in the Appendix: Supporting information.

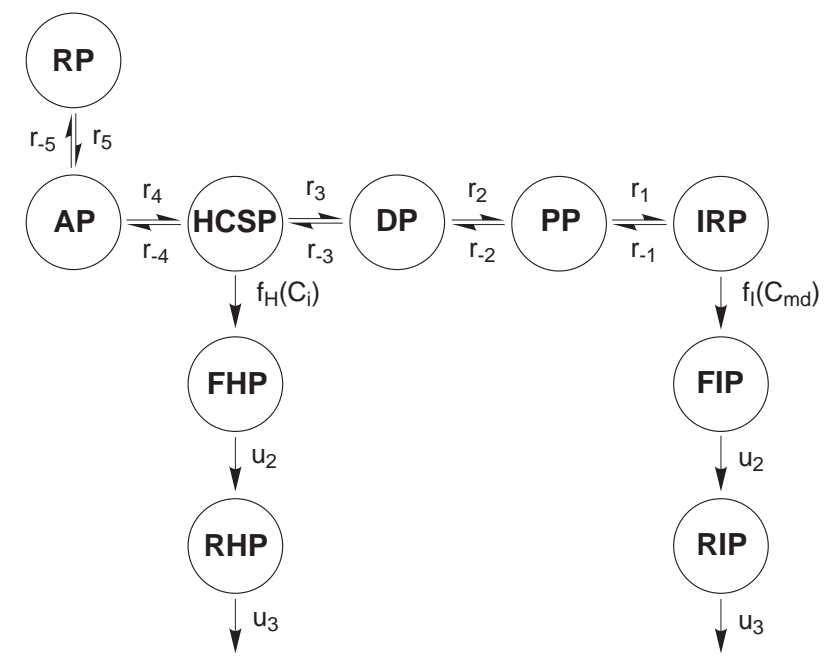

Fig. 2 Reaction overview of the model by Pedersen and Sherman [12]. The rates $r_{5}, r_{3}, r_{2}$ and $f_{H}\left(C_{i}\right)$ are a function of cytosolic calcium $C_{i}$ and the rate $f_{I}\left(C_{m d}\right)$ is the function of microdomain calcium $C_{m d}$.

The Pedersen-Sherman vesicle model is a theoretical model of granule secretion, that is, the existence of these several pools is inferred on the basis of model calibration to experimental data. The model compels an exercise to detect the molecular identity of these pools experimentally. However, an attempt to study the microscopic nature of secretion with the model will necessarily have to contend with two difficulties: (i) The granular nature of vesicles, and (ii) the stochasticity of transitions events and secretion. In the present work 
we study both of these aspects of the Pedersen-Sherman model: We construct a model equivalent to [12] with integral numbers of granules in the various pools, and in addition, reactions are determined stochastically as well.

Insulin secretion is well known to follow a two-phase pattern: a sharp, highamplitude rapid release phase that lasts about 5 minutes, and a longer, more sustained slow phase that develops over an hour. Different mechanisms have been suggested for the two phases. In one theory, the first phase is large due to exocytosis of the already docked granules and the second phase secretion is relatively flat and rises with a very slow rate due to being fed from newcomer granules $[3,6]$. Another line of experimental evidence indicates that two different releasing pools with different calcium sensitivities exist $[18,19]$. Pedersen and Sherman propose that including a pool with a high calcium sensitivity away from the L-type calcium channels leads to the newcomer granules participating in the second phase of insulin secretion $[9,8]$. To calibrate their model Pedersen and Sherman follow the experimental protocols of Yang et al. [18, 19] in which the membrane was depolarized to $20 \mathrm{mv}$ three times for $10 \mathrm{~ms}$ to stimulate the IRP, followed by a photo elevation of $C a_{i}$ to $1.8 \mu \mathrm{M}$ to release the HCSP. Parameters in [12] were chosen to represent the pools sizes obtained by Rorsman et al. [14]. To obtain release in the model on longer timescales on the order of an hour - a burst-protocol was used: successive square pulses of membrane depolarisation from $-70 \mathrm{mV}$ to $20 \mathrm{mV}$. Thus a fast protocol was picked to study secretion on a timescale of a few seconds, and a slow protocol studied secretion over a time course of 50 minutes; the two protocols reveal the relative contributions to secretion from the RRPs.

Before experiments can be carried out to investigate the identity and dynamics of the pools anatomically, it would be useful to know if the model can be placed on a firmer theoretical basis. Since experiments are carried out in terms of individual copy numbers, a translation of the concentration values to numbers is necessary. If there were large copy numbers in the pools across multiple cells, then the average behavior of the ensemble will directly correspond to the concentrations in the Pedersen-Sherman model (that is, in the thermodynamic limit of stochastics). Experiments, however, are performed with a few islets, and pool sizes are typically only in the tens per islet. Then it is necessary to know, a priori, minimum numbers of islets necessary to conduct experiments such that the deviation from the asymptotic scenario can be treated as relatively small. In a hand waving sense, tens of thousands of copy numbers are required in order to be able to safely ignore fluctuations in a simulation. To the best of our knowledge such estimates not yet been constructed for this system: There is no way to know if fluctuations are an important consideration for extending the Pedersen-Sherman model to experiments.

Further, for fitting models to experimental data it is imperative to know what variance to expect in the measured values. When the conditions of the Central Limit Theorem are satisfied $[4,17]$, variance typically scales as $1 / \sqrt{N}$ with sample size, N. However as we shall show below that this system behaves in a Poissonian fashion instead, and as such variance is equal to the mean regardless of the sample size. That is, when the size of the variation is equal to 
the size of signal itself it becomes challenging to pick out an average response especially from a few cells. Moreover, the variance cannot, a priori, be expected to decrease with the addition of a few more cells. Thus it is not clear how many experiments (i.e. how many cells) are required in order to obtain a reliable estimate of mean behavior (and variance as well).

Our stochastic simulations that track the copy numbers of vesicles reinforce the continuum-based Pedersen-Sherman model. It validates the mean behavior of the deterministic model as an average over multiple cells. Further, the stochastic simulations give a sense of the variance to be expected in secretion from individual cells. Our numerical results also demonstrate that the mean behaviour of the Pedersen-Sherman model is recovered over a thousand cells.

In the chemical reaction system when the rates are constant and do not depend on time, it is well known that using the Gillespie algorithm the average over several stochastic simulations is the deterministic solution. In [15] it has been shown that this holds true for the case with time-dependent rates as well.

In the insulin secretion granule pools model, as cytosolic calcium and microdomain calcium change with time, the reaction rates of the pool are time dependent. We propose to check numerically that using the Gillespie stochastic simulation algorithm on this model, the mean over several stochastic simulations is same as the deterministic solution. In fact because the Poisson process is involved, the variance should be equal to the mean as well.

\section{The hybrid Gillespie algorithm with time-dependent rates}

The granule pools are stimulated by an influx of calcium through L-type calcium channels; the model responds to both microdomain and cytosolic calcium rises. Calcium time courses are prescribed in the model, and the rate constants that are sensitive to calcium determine propensities of the reactions in a timevarying fashion. We therefore adopt a hybrid Gillespie SSA, that is, using ODEs to implement the choice of next reaction in the stochastic equations. This algorithm is a modification of the classical Gillespie SSA [5] to include time-dependent propensities, as explained in Fig. 3.

We note that although there are several software packages that allow for stochastic simulations, such packages as COPASI [7] typically cannot solve coupled ODE-SSA simulations. The modification of the SSA that accommodates time varying propensities is as follows. In the Gillespie SSA the next reaction time is exponentially distributed with a rate parameter equal to the

total propensity, $p_{0}=\sum_{k=1}^{M} p_{k}$ of $M$ reactions. The hybrid Gillespie algorithm replaces the constant propensities $p_{k}$ with time-varying propensities $p_{k}(t)$. In the Gillespie SSA the time of the next reaction, $t_{\text {next }}$, is distributed exponentially, that is, $e^{-p_{0} t_{n e x t}}$. The hybrid Gillespie algorithm accounts for timedependent propensities by replacing the $t_{n e x t}$ distribution as $e^{-\int_{0}^{t_{n e x t}} p_{0}(t) d t}$ [1]. As in the insulin granule pools model some of the rates are dependent on the time varying calcium as in Eqs. (22) and (23) in Appendix: Supporting 
Information. We therefore use hybrid Gillespie SSA to carry out the stochastic simulations.

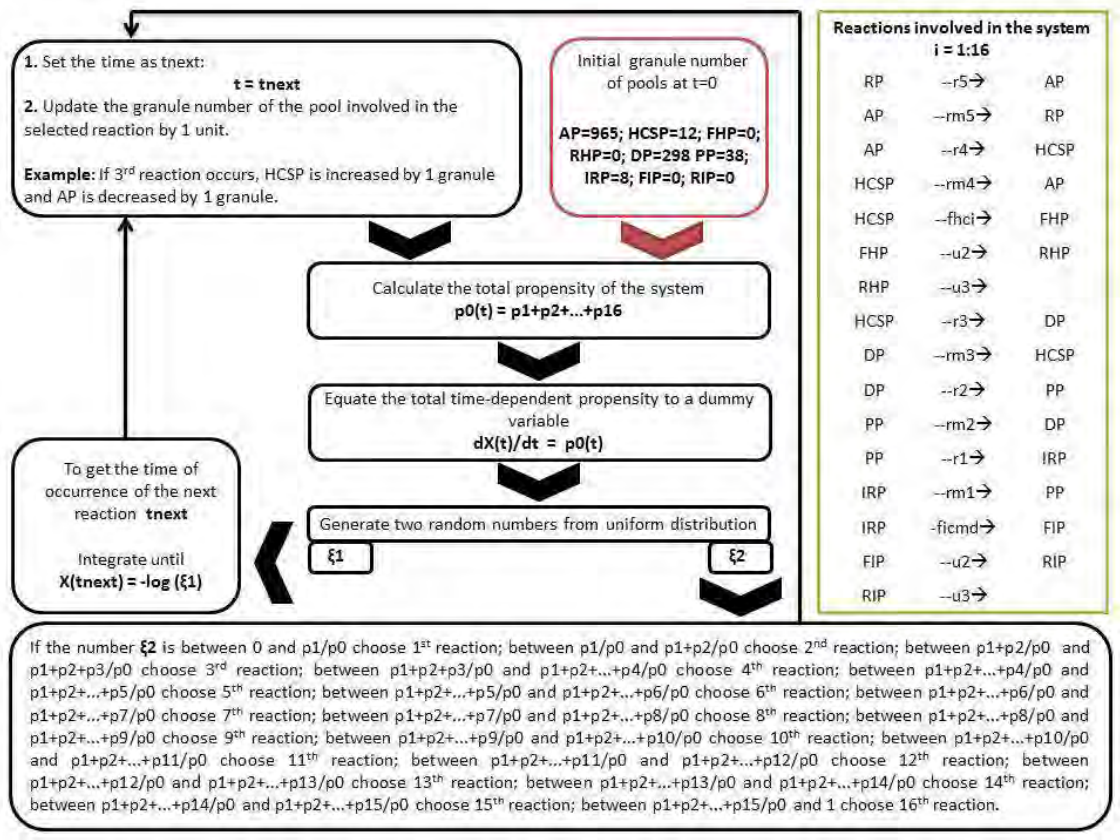

Fig. 3 Flowchart representing the steps involved in hybrid Gillespie SSA. At $t=0$, initial granule number of the pools is defined (red box). Then, total propensity is calculated corresponding to the reactions taking place in the system (green box), which is time-dependent. Later, two random numbers $\xi_{1}$ and $\xi_{2}$ are generated from uniform distribution, $\xi_{1}$ is used to calculate the time of occurrence of the next reaction and $\xi_{2}$ is used to select the reaction occurring at time of occurrence of the next reaction. The granule numbers of the pools involved in the selected reaction are updated and the process is repeated.

The pseudocode for the hybrid Gillespie SSA is as follows:

1. Compute the total propensity as

$$
p_{0}(t)=\sum_{k=1}^{M} p_{k}(t)
$$

where the index $k$ parameterizes the $M$ reactions involved, $p_{k}(t)$ is the time-varying reaction rate of the $k$ th reaction.

2. Generate two uniformly distributed random numbers, $\xi_{1}, \xi_{2} \in U[0,1]$.

3. Assume a reaction has just taken place. Initiate a differential equation equating a dummy variable to the total propensity, $\frac{d X(t)}{d t}=p_{0}(t)$ together with $X(0)=0$.

4. Integrate until $X(\tau)=-\ln \xi_{1}$.

5. Update the species involved in the reaction using $\xi_{2}$ identically as in SSA. 
We note that step (3) calls for integration of the time-varying total propensity. In the simulations below we use a simple Euler scheme with a small time step to capture stopping events with a reasonable accuracy. The algorithm can probably be investigated for more efficient integrators, which may in fact work well with additional optimizations such as tau-leaping [13]; we do not pursue these directions further in this paper.

\section{Results}

Pedersen and Sherman [12] describe two different stimulation protocols used to extract information about secretions responses from the pools. We term these the "fast" and "slow" protocols: the fast protocol is best suited for studying the secretion response over 10 seconds, while the slow protocol exacts longer time responses over 50 minutes. We first discuss the fast protocol, and will consider the slow protocol in the following sections. An islet is typically comprised of about a 1000 beta-cells. Each stochastic run corresponds to the behavior of a single cell. Here we would like to ask how many islets are expected to be required for experiments? That is, how many runs does it take for the stochastic simulations to agree, in the mean sense, with the deterministic model of Pedersen and Sherman. We calculate the mean and variance for both the protocols, and see that the mean and the variance agree well around 1000 simulations. The reason that the variance is higher for the large pools is because the mean is higher as well, and for small pools the variance is lower because the mean is lower, refer Appendix: Reservoir coupling induces variance.

\subsection{Fast responses to calcium pulses}

When simulating the model for the fast protocol as defined by Pedersen and Sherman in [12], we have taken microdomain calcium $C_{m d}$ and cytosolic calcium $C_{i}$ to be close fits to the calcium traces solved via ODEs in [12]. Simulating with the Pedersen-Sherman calcium equations, the stochastic simulations were not tracking the sudden changes in the IRP due to the microdomain calcium. The sudden peaks in $C_{m d}$ were not tracked by the SSA. This is why, we use the fits of the equations with broader pulses. We have used the MATLAB fit tool to fit the solution of the calcium ODEs as defined by Pedersen and Sherman in [12]. $C_{m d}$ is composed of three thin square pulses at $0.1 \mathrm{sec}, 0.2$ sec and 0.3 seconds of width 0.01 seconds as shown in Fig. 4(a). $C_{i}$ is held at steady-state until a sudden rise to $2 \mu M$ at 0.5 seconds depicting a flash release as shown in Fig. 4(b). The corresponding fit equations are as mentioned in Appendix: Supporting information, Eqs. (24) and (25). 


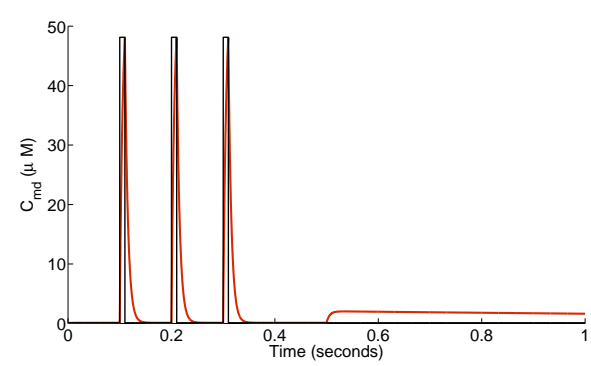

(a) Microdomain calcium

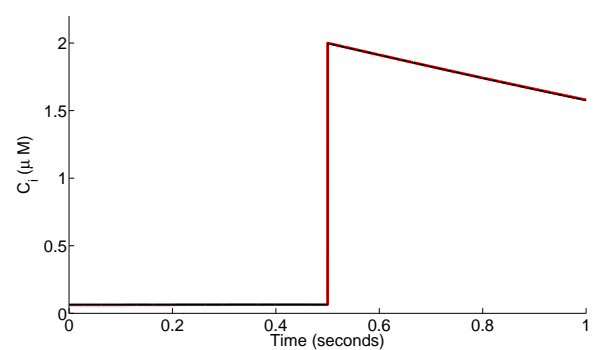

(b) Cytosolic calcium

Fig. 4 The fast stimulation protocol. Calcium concentration curves (red) compared with the fits of the calcium concentration curves provided in Eqs. (24) and (25). Calcium concentrations $(\mu M)$ are plotted versus time used to detect fast responses of the model. (a) Microdomain calcium is approximated as a function with 3 square pulses at $\mathrm{t}=0.1 \mathrm{~s}, 0.2$ $\mathrm{s}$ and $0.3 \mathrm{~s}$ and (b) Cytosolic calcium is a function with sudden rise of concentration at $\mathrm{t}$ $=0.5 \mathrm{~s}$ to depict flash release of calcium and closely overlaps with the cytosolic calcium defined by Pedersen and Sherman.

We show the mean and variance over 1000 stochastic runs compared to the deterministic solution for all the nine pools corresponding to the fast protocol, shown in the figures below: 


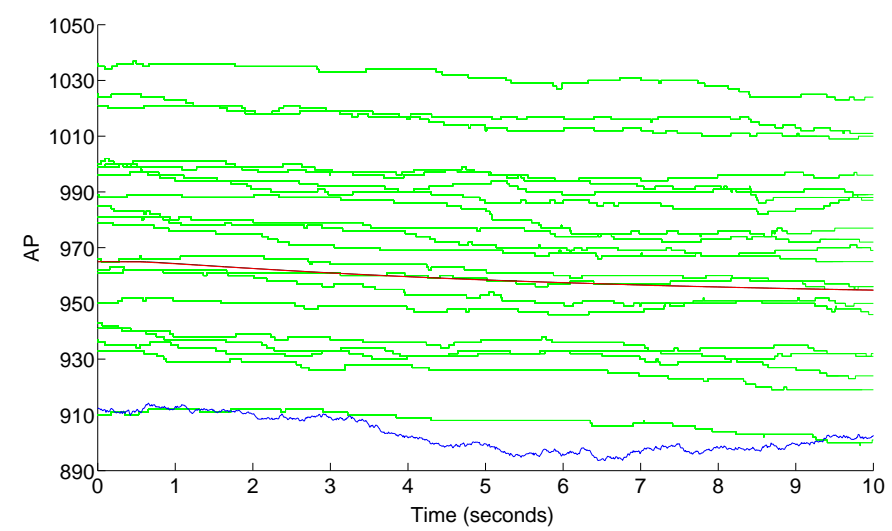

(a) AP

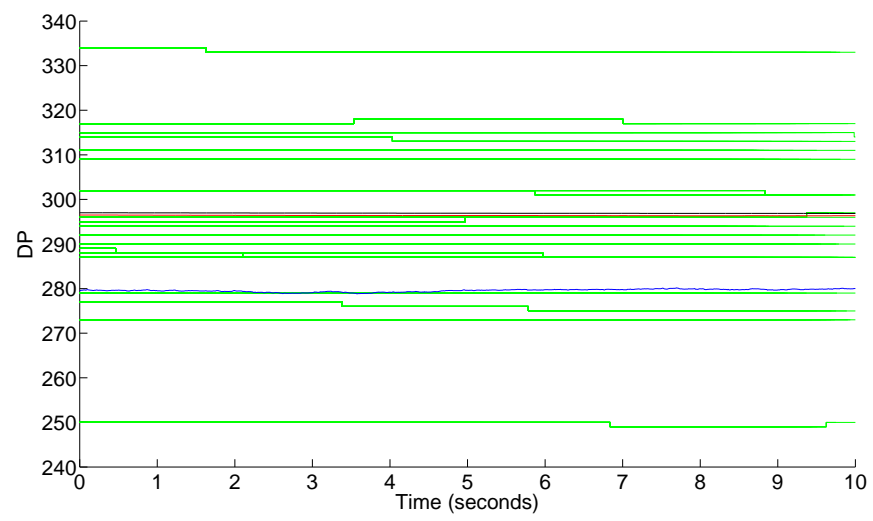

(b) DP

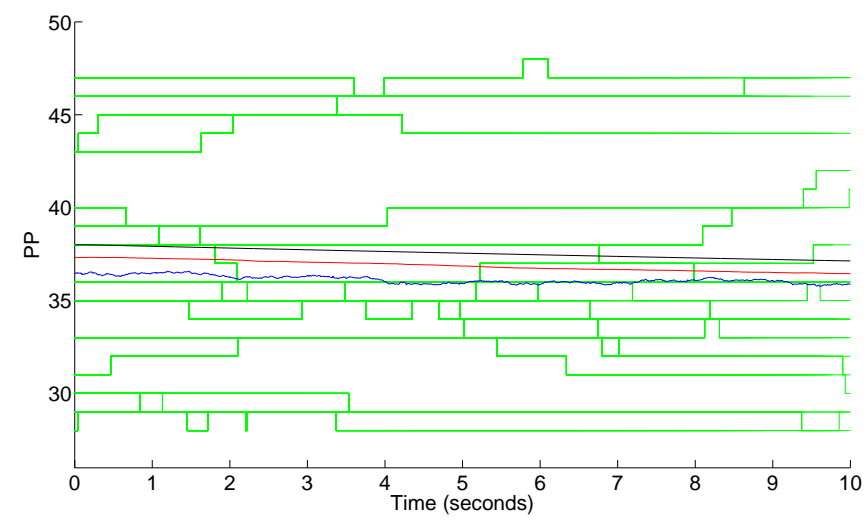

(c) PP

Fig. 5 Figures (a)-(c) are the almost docked pool (AP), docked pool (DP) and primed pool (PP), respectively, simulated with the fast protocol shown in Fig. 4 for 10 seconds. These are large pools and do not show an appreciable depletion over fast time scales. 20 stochastic runs (green) are overlaid on the deterministic solution (black). The mean (red) of AP, DP and PP over 1000 runs and variance (blue) are seen to lie close together. 


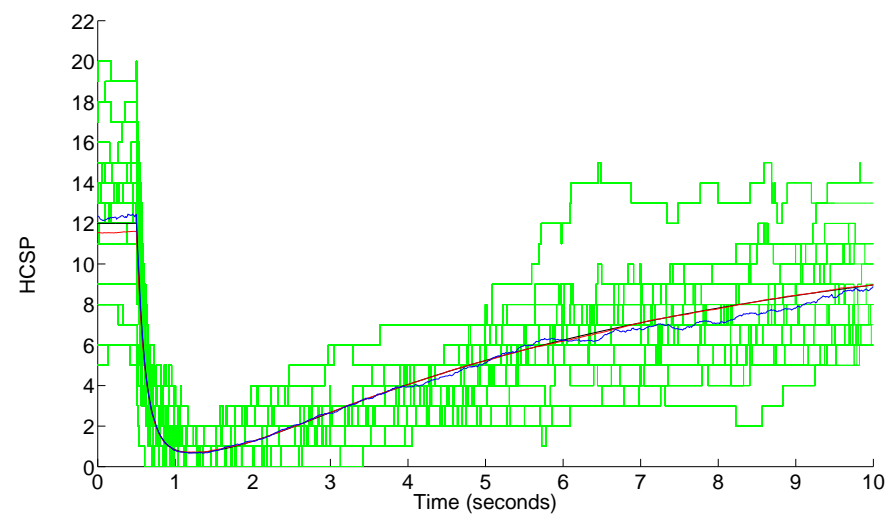

(a) HCSP

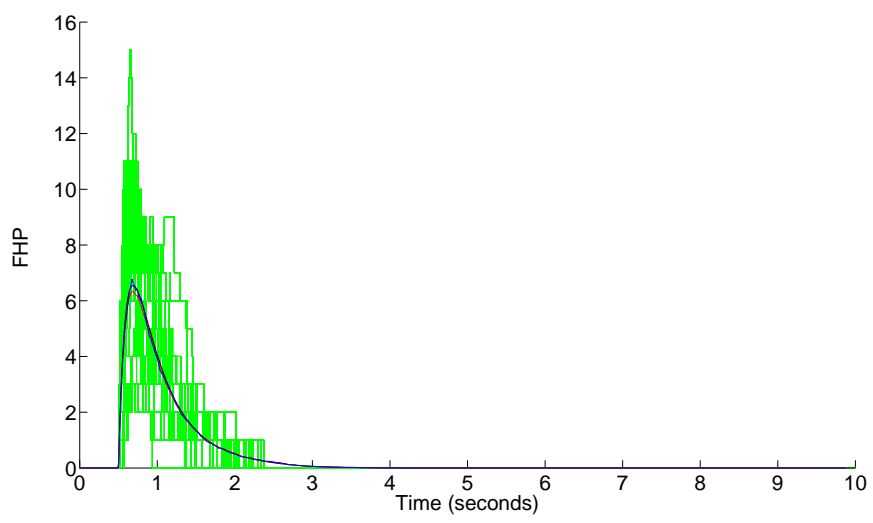

(b) FHP

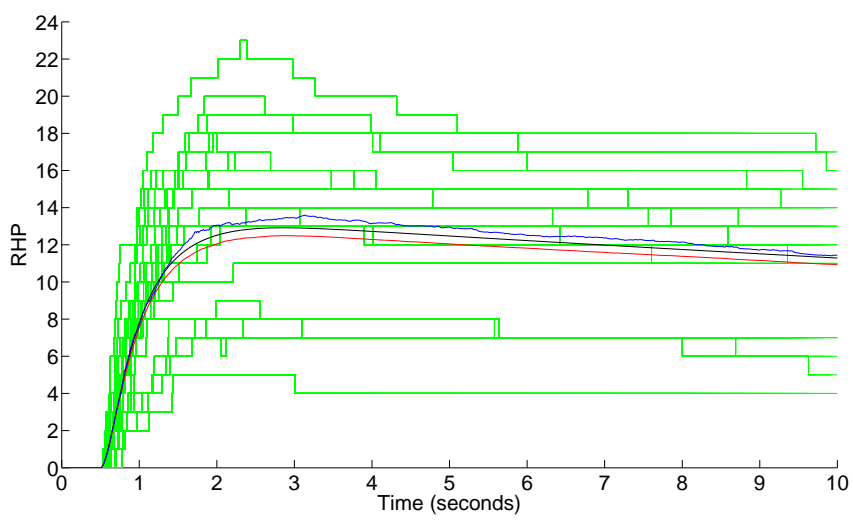

(c) RHP

Fig. 6 Figures (a)-(c) are the highly calcium sensitive pool (HCSP), its fusion (FHP)and releasing pool (RHP), respectively, simulated with the fast protocol shown in Fig. 4 for 10 seconds. The mean (red) of HCSP, FHP and RHP over 1000 runs and variance (blue) are seen to lie close to each other. 20 stochastic runs (green) and the deterministic solution (black) are also overlaid. The HCSP pool responds to a rise in cytosolic calcium $C_{i}$ at $\mathrm{t}=$ 0.5 seconds, but is largely insensitive to microdomain calcium. 


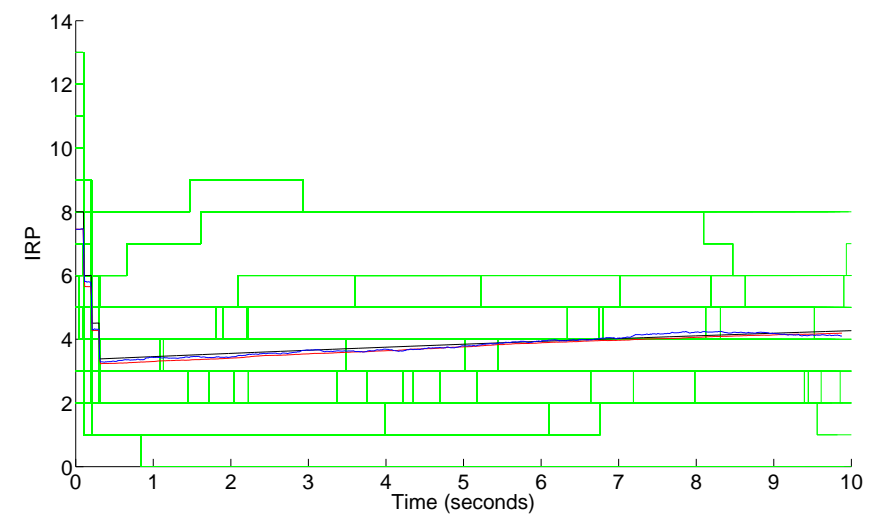

(a) IRP

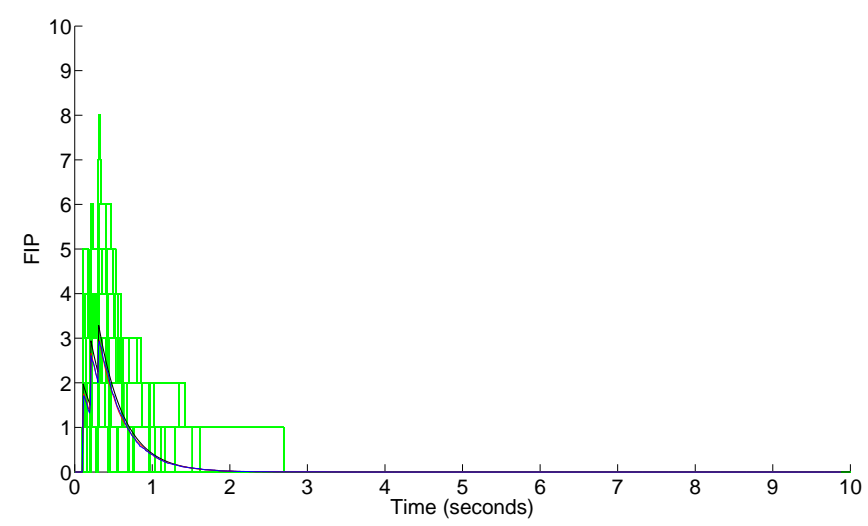

(b) FIP

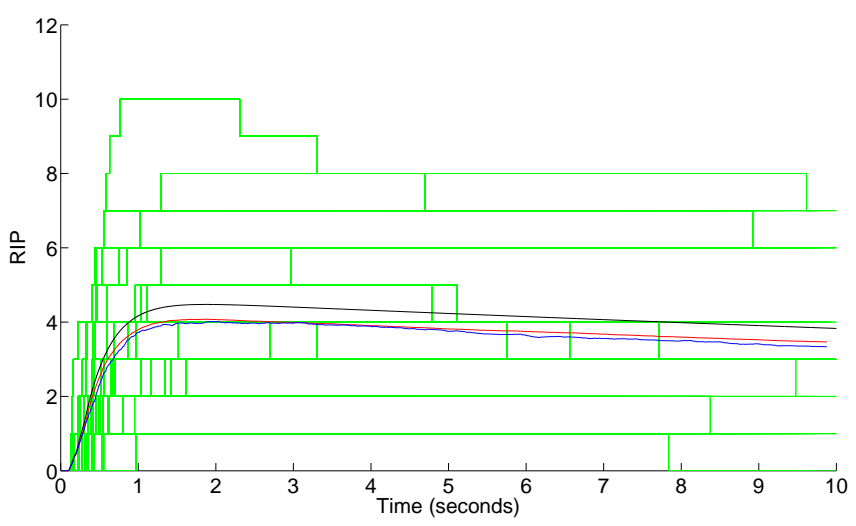

(c) RIP

Fig. 7 Figures (a)-(c) are the immediately releasable pool (IRP), its fusion (FIP) and releasing pool (RIP), respectively, simulated with the fast protocol shown in Fig. 4 for 10 seconds. The mean (red) of IRP, FIP and RIP over 1000 runs and variance (blue) are seen to lie close to each other. Overlaid are 20 stochastic runs (green) and the deterministic solution (black). The IRP chain is sensitive to the three pulses of $C_{m d}$ but not to $C_{i}$. IRP is only slowly replenished from DP and PP over ten seconds. 
In figures (5-7) we compute the mean (red) and variance (blue) over 1000 stochastic simulations with the fast protocol up to 10 seconds. To solve the ODEs involved in the Hybrid SSA, an Euler method was used with a timestep of $10^{-4}$. Fig. 7 shows that the IRP chain detects the three square pulses of the microdomain calcium at $0.1,0.2$ and 0.3 seconds due to a proximity to the L-type calcium channels. The HCSP pool detects the rise in the cytosolic calcium photo released at 0.5 seconds, Fig. $(5,6)$ but responds to microdomain calcium to a very small extent. Therefore, first phase insulin secretion is largely due to RIP (the releasing pool of IRP) and the second phase is due to RHP (that releasing pool of HCSP) as described in [12].

The average over 1000 runs of a stochastic model shows good agreement with the deterministic curves (black). This suggests that the Pedersen-Sherman model denotes the average behavior of roughly about one islet. Notice also the variance in individual runs (20 such trajectories are overlaid in green) is or the same order as the mean; an average variance computed from the 1000 runs (blue traces) also confirm the theoretical relationship expected of this system, namely variance is equal to the mean. We therefore confirm that the results of the Pedersen-Sherman deterministic concentrations for the fast protocol can be interpreted as the mean of at least 1000 stochastic simulations.

\subsection{Slow responses to calcium pulses}

In this section we discuss the slow protocol described by Pedersen and Sherman [12]. In the slow protocol in order to simulate the glucose stimulation the rate of mobilization of granules from RP to AP is augmented by a factor, Gluc:

$$
r_{5}=\text { Gluc } r_{50} \frac{C_{i}}{C_{i}+K p},
$$

where,

$$
\text { Gluc }= \begin{cases}1 & \text { for } 0 \leq t<10 \\ 3 & \text { for } t \geq 10 .\end{cases}
$$

$C_{m d}$ and $C_{i}$ are fit to the calcium traces in [12], shown in Fig. 8. The fits closely trace the calcium concentrations of Pedersen and Sherman in [12]. Hence, we use the fitted functions instead of computing $C_{i}$ and $C_{m d}$ in order to save on computational costs involved in solving the full calcium differential equations, especially since small time steps would need to be used to ensure accurate solutions. Microdomain calcium $C_{m d}$ is $263.5 \mu M$ for $t<90$ seconds and switches between the values $0.1132 \mu M$ and $263.5 \mu M$ at 30 second intervals for 3000 seconds as shown in Fig. 8(a). Similarly, cytosolic calcium $C_{i}$ switches at 30 seconds intervals for 3000 seconds as show in Fig. 8(b). The $C_{i}$ fit equation is mentioned in Appendix: Supporting information, Eq. 26 


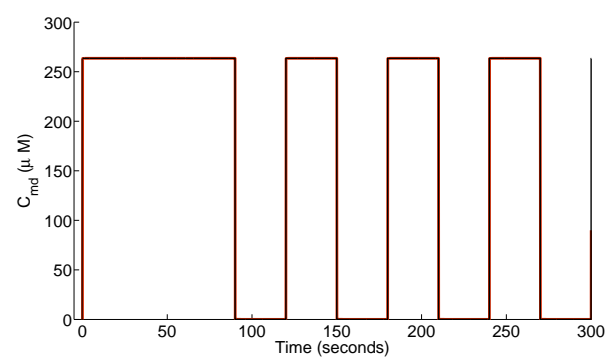

(a) Microdomain calcium

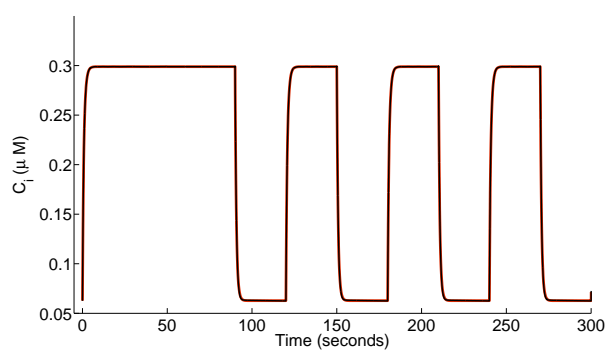

(b) Cytosolic calcium

Fig. 8 The slow stimulation protocol induced by a burst-like depolarisation pattern. The calcium trace in the deterministic model follows voltage switches (not shown), up from -70 $\mathrm{mV}$ to $-20 \mathrm{mV}$ at $\mathrm{t}=0,120,180,240$ and so on up to 3000 seconds and down from $-20 \mathrm{mV}$ to $-70 \mathrm{mV}$ at $\mathrm{t}=90,150,210$ and so on up to 2970 seconds [19,12]. Fitted microdomain and cytosolic calcium (in $\mu M$ ) (black) are shown for the first 300 seconds, and closely approximate the curves in [12] (red).

We show the mean and variance over 1000 stochastic simulations compared to the deterministic results for the slow protocol in [12]. The individual stochastic simulation solutions are not shown for the slow protocol. The figures corresponding to the stochastic simulation for the slow protocol are as shown below: 


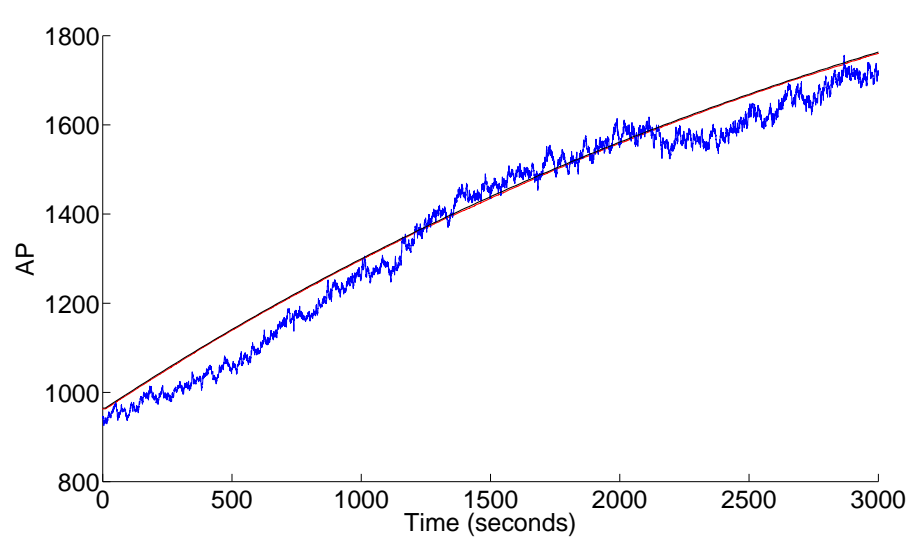

(a) AP

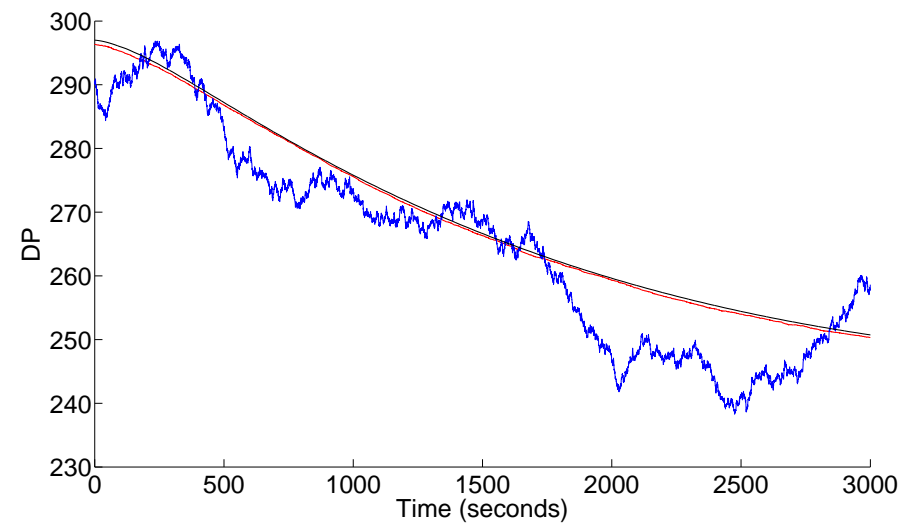

(b) DP

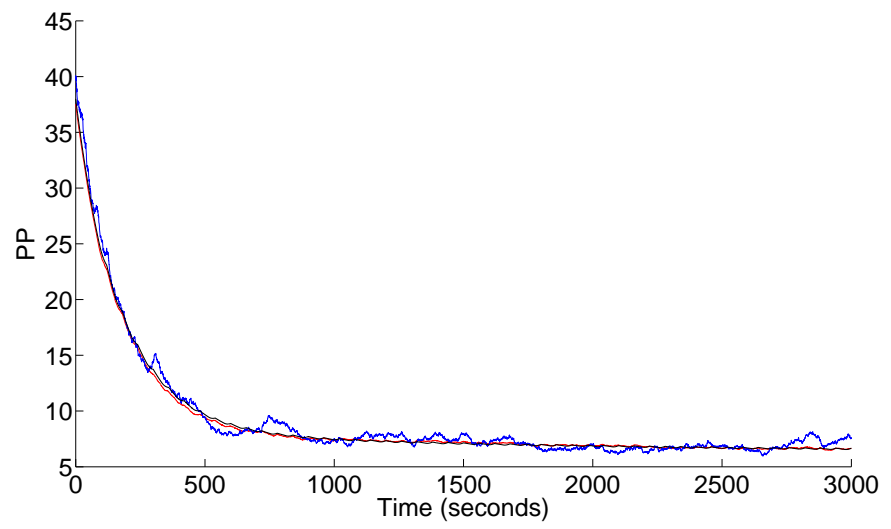

(c) PP

Fig. 9 Figures (a)-(c) are the almost docked pool (AP), docked pool (DP) and primed pool (PP), respectively, simulated with the slow protocol shown in Fig. 8 over 3000 seconds. These large pools are influenced by an increased flux of granules from RP by glucose amplification, Eq. (2), and show an appreciable change over these long timescales. The mean (red) and variance (blue) have converged close to the deterministic solution (black) for 1000 cells. 


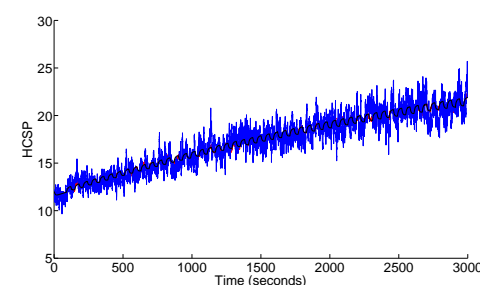

(a) HCSP

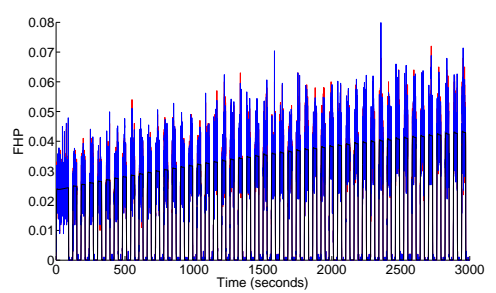

(c) FHP

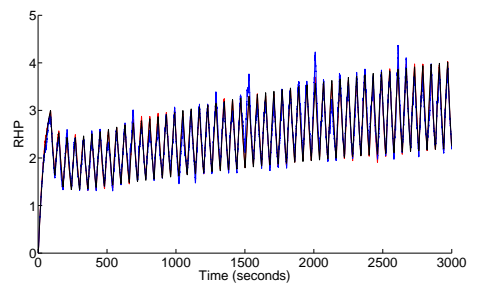

(e) RHP

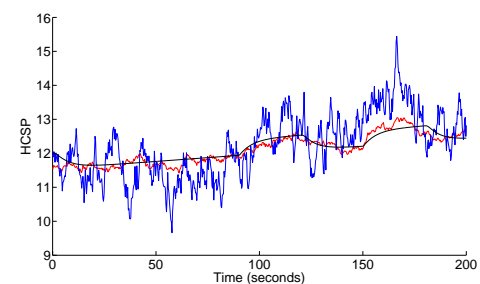

(b) HCSP; first 200s

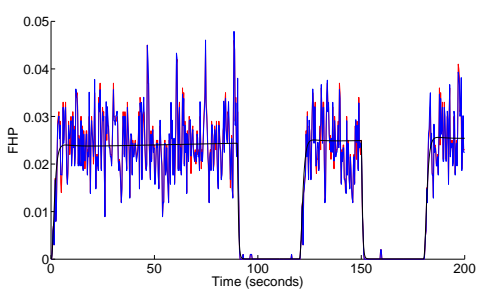

(d) FHP; first 200s

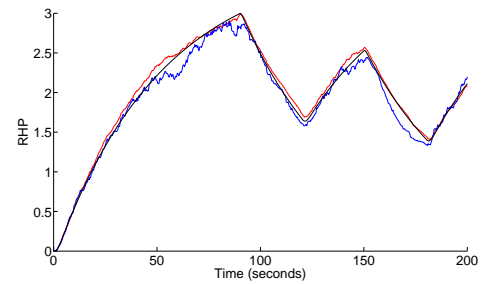

(f) RHP; first $200 \mathrm{~s}$

Fig. 10 Figures (a)-(c) are the highly calcium sensitive pool (HCSP), its fusion (FHP)and releasing pool (RHP), respectively, stimulated with the slow depolarisation protocol shown in Fig. 8 over 3000 seconds. (b), (d) and (f) are the zoomed views of HCSP, FHP and RHP, respectively up to 200 seconds. The mean (red) of HCSP, FHP and RHP over 1000 stochastic runs and variance (blue) are seen to lie close to the deterministic solution. The HCSP pool responds actively to the cytosolic calcium, $C_{i}$, and is largely insensitive to microdomain calcium, $C_{m d}$. Notice the slow overall increase in the HCSP is the major component of second phase insulin secretion. 


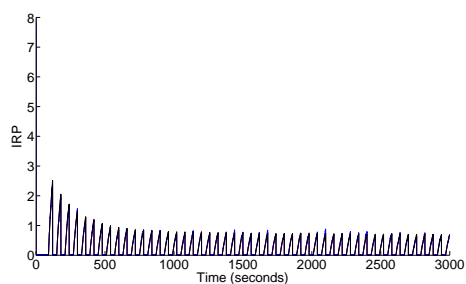

(a) IRP

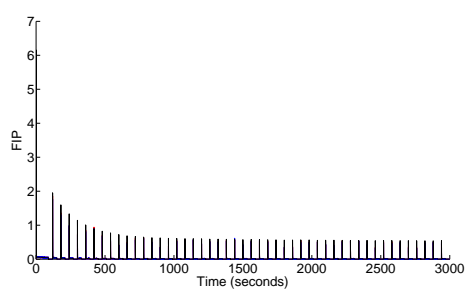

(c) FIP

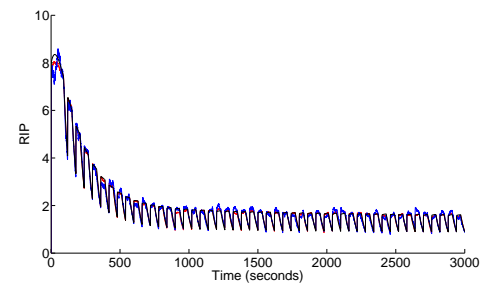

(e) RIP

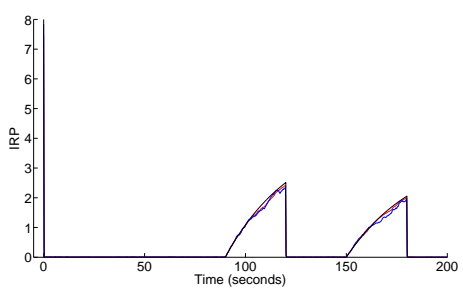

(b) IRP; first 200s

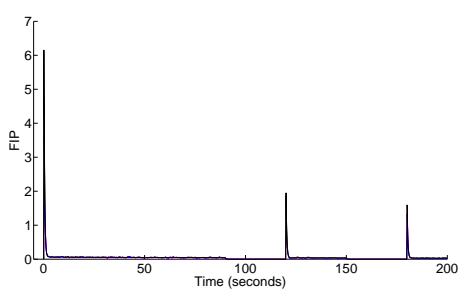

(d) FIP; first 200s

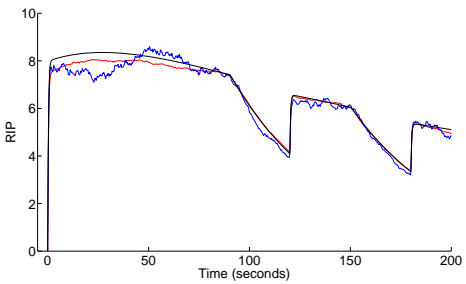

(f) RIP; first 200s

Fig. 11 Figures (a)-(c) are the immediately releasable pool (IRP), its fusion (FIP) and releasing pool (RIP), respectively, stimulated with the slow depolarisation protocol shown in Fig. 8 over 3000 seconds. (b), (d) and (f) are the zoomed view of IRP, FIP and RIP, respectively for the first 200 seconds. The mean (red) of IRP, FIP and RIP over 1000 stochastic simulations and variance (blue) are seen to lie close to the deterministic solution. The IRP chain is sensitive to the microdomain calcium, $C_{m d}$, and much less to cytosolic calcium, $C_{i}$. Notice that secretion is heavy in the first phase.

In figures (9-11) we compute the mean (red) and variance (blue) over 1000 stochastic simulations with the slow protocol for 3000 seconds. The Euler time step was taken to be $10^{-4}$. Fig. 9 shows the mean and variance for the large pools AP,DP and PP which are influenced by an increased flux of granules from RP by glucose amplification. These pools show an appreciable change over the long timescale. The highly calcium sensitive pool (HCSP), its fusion pool (FHP) and its releasing pool (RHP) are shown in Fig. 10. These pools respond mainly to the cytosolic calcium $\left(C_{i}\right)$ and are largely insensitive to the microdomain calcium $\left(C_{m d}\right)$. The increase in the HCSP is the major component of second phase insulin secretion. Fig. 11 shown immediately releasable pool (IRP), its fusion pool (FIP) and releasing pool (RIP). These pools are mainly sensitive to $C_{m d}$ and much less to $C_{i}$. Secretion from the IRP is dominant in the first phase. 
In the case with slow protocol also, we show that mean over 1000 stochastic simulations shows good agreement with the deterministic results in [12]. Also, the average variance computed from the 1000 runs (blue traces) agrees with the theoretical relationship expected of this system, namely variance is equal to the mean. We therefore confirm that the results of the PedersenSherman deterministic concentrations for the slow protocol can be interpreted as the mean of 1000 stochastic simulations as also shown for the fast protocol simulations in the above section.

Therefore, we can confidently state that the mean over around 1000 stochastic simulations or one islet (1000 cells) is enough to trace the Pedersen-Sherman deterministic results [12].

\section{Discussion}

The Pedersen-Sherman model is currently the most comprehensive insulin secretion model which is able to explain an impressive array of experimental observations $[10,11,16]$. However, before it can be successfully used together with experiments to determine the molecular identities of the granule pools, a few questions need to be addressed of the model itself, namely the consideration of discrete vesicle numbers and the stochasticity of transition events. From an experimentation point of view, it is crucial to know the coarseness with which measurements need to be made, that is, whether to seek concentrations or discrete particles. Our model assumes each pool is comprised of integral numbers of granules, and estimates the mean behaviour of stochastic runs over multiple beta-cells. The current model is thus able to inform instrumentation in advising the technique appropriate to detection of each pool: for the large pools AP, DP and PP, modeling techniques sensitive only to mass concentrations are likely to be adequate, while for the smaller pools, the HCSP and IRP chains, algorithm that explicitly tracks discrete numbers would probably be best effective. When the experimental technique used is also sensitive to fluctuations (such as atomic force microscopy or total internal reflection microscopy), we note that variances in the pool sizes are expected to be on the order of the mean copy numbers.

Finally, in order to use the Pedersen-Sherman model to study mean behaviour, our simulations have indicated that around thousand beta-cells are enough to get good expected values of the means. Thus, one islet is ought to be sufficient to perform experiments with. 


\section{A Appendix : Supporting Information}

\section{A.1 Equations}

Reactions in the stochastic model, Fig. 2, correspond to the differential equations in [12]. Initial conditions are shown in Table A.2, and the parameters are given in Table A.3.

$$
\begin{aligned}
I R P^{\prime} & =\left(r_{1} P P-r_{-1} I R P-f_{I}\left(C_{m d}\right) I R P\right) \\
P P^{\prime} & =\left(r_{-1} I R P-\left(r_{1}+r_{-2}\right) P P+r_{2} D P\right) \\
D P^{\prime} & =\left(r_{3} H C S P+r_{-2} P P-\left(r_{-3}+r_{2}\right) D P\right) \\
A P^{\prime} & =\left(r_{5}-r_{-5} A P-r_{4} A P+r_{-4} H C S P\right) \\
H C S P^{\prime} & =\left(r_{4} A P-\left(r_{-4}+r_{3}\right) H C S P+r_{-3} D P-f_{H}\left(C_{i}\right) H C S P\right) \\
F I P^{\prime} & =\left(f_{I}\left(C_{m d}\right) I R P-u_{2} F I P\right) \\
R I P^{\prime} & =\left(u_{2} F I P-u_{3} R I P\right) \\
F H P^{\prime} & =\left(f_{H}\left(C_{i}\right) H C S P-u_{2} F H P\right) \\
R H P^{\prime} & =\left(u_{2} F H P-u_{3} R H P\right)
\end{aligned}
$$

where $r_{2}=r_{20} \frac{C_{i}}{C_{i}+K p_{2}} ; r_{3}=r_{30} \frac{C_{i}}{C_{i}+K p} ; r_{5}=r_{50} \frac{C_{i}}{C_{i}+K p}$

In [12] $C_{m d}$ and $C_{i}$ are solved via the following ODEs. Molar fluxes through L-type and R-type calcium channels are defined as

$$
\begin{aligned}
& J_{L}=\alpha I_{L} / v_{m d}, \\
& J_{R}=\alpha I_{R} / v_{\text {cell }},
\end{aligned}
$$

where the respective currents are

$$
\begin{aligned}
& I_{L}=g_{L} m_{\infty}(v)\left(V-V_{C a}\right), \\
& I_{R}=g_{R} m_{\infty}(v)\left(V-V_{C a}\right),
\end{aligned}
$$

with

$$
m_{\infty}(v)=1 /\left(1+\exp \left(\left(V_{m}-V\right) / s_{m}\right)\right)
$$

Calcium pumps and calcium stores fluxes are

$$
\begin{aligned}
J_{\text {serca }} & =J_{\text {serca }}^{\max } \frac{C_{i}^{2}}{K_{\text {serca }}^{2}+C_{i}^{2}}, \\
J_{\text {pmca }} & =J_{\text {pmca }}^{\max } \frac{C_{i}}{K_{\text {pmca }}+C_{i}}, \\
J_{\text {ncx }} & =J_{\text {ncx } 0}\left(C_{i}-0.25\right) \\
L & =J_{\text {serca }}+J_{\text {pmca }}+J_{\text {ncx }}+J_{\text {leak }},
\end{aligned}
$$

Compartmental calcium equations are:

$$
\begin{aligned}
C_{m d}^{\prime} & =\left(-f_{m d} J_{L}-f_{m d} B\left(C_{m d}-C_{i}\right)\right), \\
C_{i}^{\prime} & =\left(-f_{i} J_{R}+f_{v} f_{i} B\left(C_{m d}-C_{i}\right)-f_{i} L\right),
\end{aligned}
$$

where $C_{m d}$ is microdomain calcium and $C_{i}$ is bulk cytosolic calcium concentration, measured in $\mu M$.

Instead of using the equations for calcium compartments mentioned above, we have fit $C_{m d}$ and $C_{i}$ using time-dependent functions as described below:

The fit equations corresponding to the fast protocol are as follows:

$$
\begin{aligned}
C_{m d}(t)= & 0.11+48.01 \Theta(t-0.1) \Theta(0.1+0.01-t)) \\
& +(\Theta(t-0.2) \Theta(0.2+0.01-t)) \\
& +(\Theta(t-0.3) \Theta(0.3+0.01-t)),
\end{aligned}
$$


where, $\Theta(t)$ is the Heaviside function. $C_{i}$ is held at steady-state until a sudden rise to $2 \mu M$ at 0.5 seconds following a flash release as shown in Fig. 4(b):

$$
C_{i}(t)= \begin{cases}0.06 & \text { for } t<0.5 \\ \left\{1.3 \exp \left(-((t+0.63) / 1.2)^{2}\right)\right. & \\ +\left(3.3 \times 10^{8}\right) \exp \left(-((t+1036) / 220.8)^{2}\right) & \\ \left.+1.4 \exp \left(-((t-0.47) / 2)^{2}\right)\right\} & \text { for } t \geq 0.5\end{cases}
$$

The $C_{i}$ fit equation corresponding to the slow protocol is as follows:

$$
\begin{aligned}
a(t) & =0.3 \exp \left(-((t-53.43) / 232.3)^{2}\right)+0.0048 \exp \left(-((t-5.85) / 3.02)^{2}\right) \\
& -1.3 \times 10^{10} \exp \left(-((t-(-37.3)) / 7.5)^{2}\right) \\
& -0.0028 \exp \left(-((t-13.55) / 4.3)^{2}\right)+0.0036 \exp \left(-((t-3.6) / 1.73)^{2}\right) \\
& +0.0068 \exp \left(-((t-11.81) / 6.13)^{2}\right)+0.0054 \exp \left(-((t-16.8) / 17.26)^{2}\right) \\
b(t) & =\left(5.02 \times 10^{4}\right) \exp \left(-((t+19.1) / 5.45)^{2}\right) \\
& +0.076 \exp \left(-((t-(-851.6)) / 1985)^{2}\right)
\end{aligned}
$$

$C_{i}$ is increasing at a rate $a(t)$ and decreasing with rate $b(t)$.

Fusion rates from $\operatorname{IRP}\left(f_{I}\left(C_{m d}\right)\right)$ and $\operatorname{HCSP}\left(f_{H}\left(C_{i}\right)\right)$ follow Hill functions,

$$
\begin{aligned}
f_{I}\left(C_{m d}\right) & =f_{I}^{\max } \frac{C_{m d}^{n}}{K_{I}^{n}+C_{m d}^{n}}, \\
f_{H}\left(C_{i}\right) & =f_{H}^{\max } \frac{C_{i}^{n}}{K_{H}^{n}+C_{i}^{n}}
\end{aligned}
$$

\section{A.2 Initial conditions}

Pool numbers at the beginning of the simulations were assigned from an appropriate normal distribution, $\mathrm{N}(\mu, \sqrt{\mu})$, where $\mu$ corresponds roughly to the steady-state concentration of the pool. Table A.2 shows the distributions corresponding to each pool, and the steady state values of microdomain and cytosolic calcium concentrations as well [12].

Table A.2: Initial conditions and steady state

\begin{tabular}{|c|c|}
\hline Pool & Initial condition \\
\hline IRP & $\mathrm{N}(8, \sqrt{8})$ \\
\hline PP & $\mathrm{N}(38, \sqrt{38})$ \\
\hline DP & $\mathrm{N}(298, \sqrt{298})$ \\
\hline FIP & 0 \\
\hline RIP & 0 \\
\hline AP & $\mathrm{N}(965, \sqrt{965})$ \\
\hline HCSP & $\mathrm{N}(12, \sqrt{12})$ \\
\hline FHP & 0 \\
\hline RHP & 0 \\
\hline
\end{tabular}

\begin{tabular}{|c|c|}
\hline Calcium domain & Steady state $(\mu M)$ \\
\hline$C_{m d}$ & 0.0674 \\
\hline$C_{i}$ & 0.06274 \\
\hline
\end{tabular}




\section{A.3 Parameters}

Parameters of the model are as in [12].

Table A.3: Parameters

\begin{tabular}{|c|c|c|c|}
\hline Parameter & Value & Parameter & Value \\
\hline \hline$r_{1}$ & $0.005 \mathrm{~s}^{-1}$ & $g_{L}$ & $150 \mathrm{pS}$ \\
\hline$r_{-1}$ & $0.025 \mathrm{~s}^{-1}$ & $g_{R}$ & $150 \mathrm{pS}$ \\
\hline$r_{20}$ & $0.00015 \mathrm{~s}^{-1}$ & $V_{m}$ & $-20 \mathrm{mV}$ \\
\hline$r_{-2}$ & $0.001 \mathrm{~s}^{-1}$ & $V_{C a}$ & $25 \mathrm{mV}$ \\
\hline$r_{30}$ & $0.002 \mathrm{~s}^{-1}$ & $S_{m}$ & $5 \mathrm{mV}$ \\
\hline$r_{-3}$ & $0.00007 \mathrm{~s}^{-1}$ & $J_{\text {Serca }}^{\text {max }}$ & $41 \mu M / s$ \\
\hline$r_{4}$ & $0.002 \mathrm{~s}^{-1}$ & $K_{\text {Serca }}$ & $0.27 \mu M$ \\
\hline$r_{-4}$ & $0.16 \mathrm{~s}^{-1}$ & $J_{\text {pmca }}^{\text {max }}$ & $21 \mu M / s$ \\
\hline$r_{5}$ & $0.224 \mathrm{~s}^{-1}$ & $K_{\text {pmca }}$ & $0.5 \mu M$ \\
\hline$r_{-5}$ & $0.0002 \mathrm{~s}^{-1}$ & $J_{\text {leak }}$ & $-0.94 \mu M / s$ \\
\hline$u_{1}$ & $2000 \mathrm{~s}^{-1}$ & $J_{n c x 0}$ & $18.67 \mathrm{~s}$ \\
\hline$u_{2}$ & $3 \mathrm{~s}^{-1}$ & $f_{m d}$ & 0.01 \\
\hline$u_{3}$ & $0.02 \mathrm{~s}^{-1}$ & $f_{i}$ & 0.01 \\
\hline$k_{p}$ & 0.01 & $B$ & $17250 \mathrm{~s}$ \\
\hline$k_{2}$ & 0.01 & $\alpha$ & $5.18 \times 10^{-15} \mu m o l / s / f A$ \\
\hline$f_{I}^{\text {max }}$ & $30 \mathrm{~s}^{-1}$ & $v_{c e l l}$ & $1.15 \times 10^{-12} \mathrm{pl}$ \\
\hline$K_{I}$ & $22 \mu M$ & $v_{m d}$ & $0.00385 \times 10^{-15} \mathrm{pl}$ \\
\hline$f_{H}^{\text {max }}$ & $30 \mathrm{~s}^{-1}$ & $f_{v}$ & $v_{m d} / v_{c e l l}$ \\
\hline$K_{H}$ & $2.5 \mu M$ & $n$ & 4 \\
\hline
\end{tabular}




\section{B Appendix : Reservoir coupling induces variance}

It is interesting to note that there are two major sources of variance in pool sizes. We discuss next its implications in the context of the stochastic model simulations.

Copy numbers of the pools are integral, and stochastic, as are the transitions between the states as well; hence variance is introduced into granule numbers as the system evolves. In other words, even if multiple instances of the model were each started with the same initial numbers of granules, in say AP, fluctuations would be rapidly introduced in the pools downstream (and AP as well). On the other hand, the simulations have also to account for the fact that even if the pools are at equilibrium, that is, unstimulated, fluctuations will naturally arise in the pool numbers simply from stochastic transitions between the various states. Each of these sources of variation in the pools has important implications for a correct estimation of the expected values of variance in the simulations.

In the Pedersen-Sherman model the almost-docked pool is in contact with a large reservoir-style "reserve pool", a much larger source of vesicles maintained in the Golgi body with which the AP is largely in equilibrium. (Because of this flux from the reserve pool we view this is an open system). On the other hand, if the AP were modeled as a fixed, large-sized pool with no source term, that closed system would differ from the open system in only an RP $\longleftrightarrow$ AP flux. In the Pedersen-Sherman model AP decays very slowly during exocytosis, at least over a few seconds. For modeling AP on that timescale, either an open or a closed system would appear to be very similar. Indeed, in either situation the mean sizes of all the pools are very nearly identical (simulations not shown). However, it turns out that if the pools are initiated with the same constant numbers across multiple instances of the stochastic simulations, that is, in the closed system, variance is invariably underestimated throughout time (without affecting the mean). It is therefore important to assign variance correctly when determining the initial values of pool numbers. In other words, we have to account for fluctuations in the initial states of the pools at equilibrium ${ }^{1}$, or a component of variance would be missing in the simulations.

This behavior can be demonstrated in a simple example. We compute below the expectations of mean and variance for a simple decay process, with and without coupling to a source flux.

1. A simple decay process. For a pure death process

$$
\mathrm{AP} \stackrel{k_{1}}{\longrightarrow} \phi
$$

the mean evolves as

$$
\frac{d \mathbf{E}[\mathrm{AP}]}{d t}=-k_{1} \mathbf{E}[\mathrm{AP}]
$$

and the variance can be shown to be

$$
\operatorname{Var}[\mathrm{AP}]=\mathrm{AP}(0) \mathrm{e}^{-\mathrm{k}_{1} \mathrm{t}}\left(1-\mathrm{e}^{-\mathrm{k}_{1} \mathrm{t}}\right),
$$

where $\mathbf{E}[\mathrm{AP}]$ and $\operatorname{Var}[\mathrm{AP}]$ represent the expectation value of mean, and variance of $\mathrm{AP}(\mathrm{t})$, respectively $[4,17]$. Figure 12 shows the average result of 5000 instances of this process. Notice that variance is zero in the beginning because we assume each simulation to be started with exactly the same number of $\mathrm{AP}$ particles, namely $\mathrm{AP}(0)=5$.

2. A decay process coupled to a reservoir. On the other hand, for

$$
\stackrel{k_{0}}{\longrightarrow} A P \stackrel{k_{1}}{\longrightarrow} \phi .
$$

the mean evolves as

$$
\frac{d \mathbf{E}[\mathrm{AP}]}{d t}=k_{0}-k_{1} \mathbf{E}[\mathrm{AP}]
$$

1 Of course, the open system has the additional advantageous feature that the system can asymptotically be in an equilibrium with a reservoir, while in a closed system AP would (eventually) be depleted completely. 


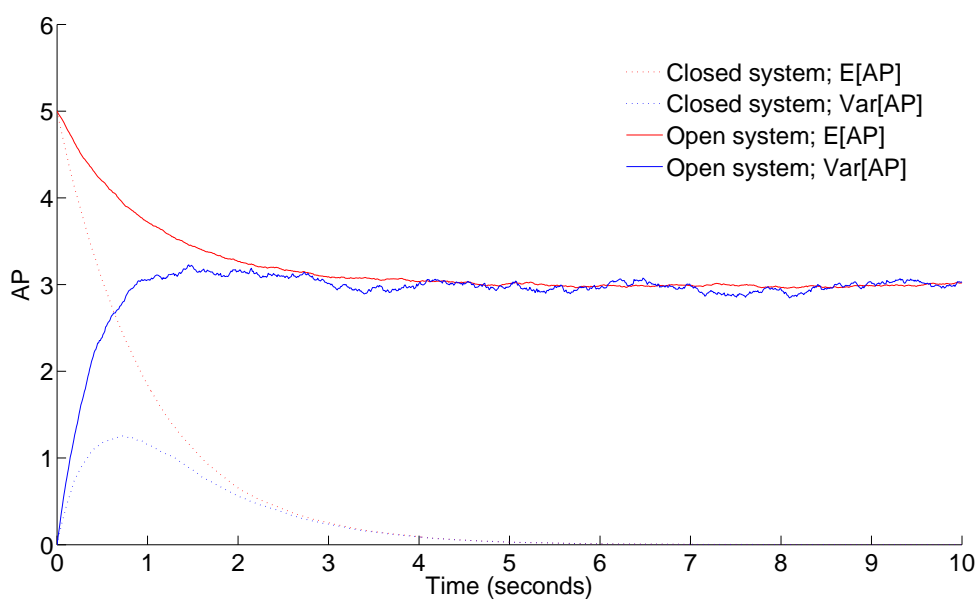

Fig. 12 The average behaviour of E[AP] and $\operatorname{Var}[\mathrm{AP}]$ over 5000 instances using Gillespie SSA. In both open and close system, in each instance $\operatorname{AP}(0)=5$ and $\operatorname{Var}[\mathrm{AP}](\mathrm{t}=0)=0$. Also the parameters $k_{0}=3$ and $k_{1}=1$. In the open system, both the mean and the variance are settling near the value $k_{0} / k_{1}$. In the closed system, $\mathrm{E}[\mathrm{AP}] \rightarrow 0$ and $\operatorname{Var}[\mathrm{AP}] \rightarrow 0$ as $\mathrm{t} \rightarrow \infty$. Notice also that if $k_{0}<<k_{1}$, the asymptotic value of $\mathbf{E}[\mathrm{AP}]$ in (32) is nearly zero as well.

and

$$
\frac{d \mathbf{E}\left[\mathrm{AP}^{2}\right]}{d t}=k_{0}-2 k_{1} \mathbf{E}\left[\mathrm{AP}^{2}\right]+\left(2 \mathrm{k}_{0}+\mathrm{k}_{1}\right) \mathbf{E}[\mathrm{AP}] .
$$

The variance can be computed from Eqs. (33) and (34); its steady-state value can be seen to satisfy $\operatorname{Var}[\mathrm{AP}]=k_{0} / k_{1}$. Also notice that, as above, $\operatorname{Var}[\mathrm{AP}(\mathrm{t}=\infty)]=\mathbf{E}[\mathrm{AP}(\mathrm{t}=$ $\infty)$ ], although only system (32) can asymptotically be at an equilibrium away from zero.

Figure 12 demonstrates that variance in the open system ought to be initialized to the steady-state mean values of the pools. For details of the initial pool sizes in the model simulations see Table A.2.

Thus variance in the pool sizes arises from two sources, only one of which is the stochasticity that arises as the system evolves. The other component that introduces variance in the system is reservoir coupling: initial fluctuations, especially in the AP pool, need to be accounted correctly before calcium stimulations can be examined in the model. 
Acknowledgements I would like to thank Dr. Pranay Goel for his constant support and guidance, Dr. Chetan Gadgil for his valuable suggestions, Shahdab Alam for helping me through early phase of my work in MATLAB and Dr. Arthur Sherman for his help with the model in [12], and for valuable discussions.

\section{References}

1. A. Alfonsi, E. Cances, G. Turinici, B. D. Ventura, and W. Huisinga. Adaptive simulation of hybrid stochastic and deterministic models for biochemical systems. ESAIM: PROCEEDINGS, 14:1-13, September 2005.

2. Y. D. Chen, S. Wang, and A. Sherman. Identifying the targets of the amplifying pathway for insulin secretion in pancreatic beta-cells by kinetic modeling of granule exocytosis. Biophys. J., 95(5):2226-2241, September 2008.

3. D. L. Curry, L. L. Bennett, and G. M. Grodsky. Dynamics of insulin secretion by the perfused rat pancreas. Endocrinology, 83(3):572-584, September 1968.

4. C.W. Gardiner. Handbook of stochastic methods for physics, chemistry, and the natural sciences. Springer series in synergetics. Springer, second edition, 1994.

5. D. T. Gillespie. Exact stochastic simulation of coupled chemical reactions. The Journal of Physical Chemistry, 81(25):2340-2361, 1977.

6. J. C. Henquin, M. Nenquin, P. Stiernet, and B. Ahren. In vivo and in vitro glucoseinduced biphasic insulin secretion in the mouse: pattern and role of cytoplasmic $\mathrm{Ca} 2+$ and amplification signals in beta-cells. Diabetes, 55(2):441-451, Feburary 2006

7. S. Hoops, S. Sahle, R. Gauges, C. Lee, J. Pahle, N. Simus, M. Singhal, L. Xu, P. Mendes, and U. Kummer. COPASI-a COmplex PAthway SImulator. Bioinformatics, 22(24):3067-3074, December 2006.

8. K. Kasai, T. Fujita, H. Gomi, and T. Izumi. Docking is not a prerequisite but a temporal constraint for fusion of secretory granules. Traffic, 9:1191-1203, July 2008.

9. M. Ohara-Imaizumi, C. Nishiwaki, T. Kikuta, S. Nagai, Y. Nakamichi, and S. Nagamatsu. TIRF imaging of docking and fusion of single insulin granule motion in primary rat pancreatic beta-cells: different behaviour of granule motion between normal and Goto-Kakizaki diabetic rat beta-cells. Biochem. J., 381(Pt 1):13-18, July 2004.

10. M. G. Pedersen. Insulin secretory granules enter a highly calcium-sensitive state following palmitate-induced dissociation from calcium channels - a theoretical study. Journal of Neuroendocrinology, 22(12):1315-1324, December 2010.

11. M. G. Pedersen. On depolarization-evoked exocytosis as a function of calcium entry: possibilities and pitfalls. Biophys. J., 101(4):793-802, August 2011.

12. M. G. Pedersen and A. Sherman. Newcomer insulin secretory granules as a highly calcium-sensitive pool. Proc. Natl. Acad. Sci. U.S.A., 106(18):7432-7436, May 2009.

13. M. Rathinam, L. R. Petzold, Y. Cao, and D. T. Gillespie. Stiffness in stochastic chemically reacting systems: The implicit tau-leaping method. The Journal of Chemical Physics, 119:12784-12794, December 2003.

14. P. Rorsman and E. Renstrom. Insulin granule dynamics in pancreatic beta cells. Diabetologia, 46(8):1029-1045, August 2003.

15. V. Shahrezaei, J. F. Ollivier, and P. S. Swain. Colored extrinsic fluctuations and stochastic gene expression. Mol. Syst. Biol., 4:196, May 2008.

16. K. Tsaneva-Atanasova, H. M. Osinga, J. Tabak, and M. G. Pedersen. Modeling mechanisms of cell secretion. Acta Biotheor., 58(4):315-327, December 2010.

17. N. G. Van Kampen. Stochastic Processes in Physics and Chemistry, Third Edition (North-Holland Personal Library). Third edition, 2007.

18. Q. F. Wan, Y. Dong, H. Yang, X. Lou, J. Ding, and T. Xu. Protein kinase activation increases insulin secretion by sensitizing the secretory machinery to Ca2+. J. Gen. Physiol., 124(6):653-662, December 2004.

19. Y. Yang and K. D. Gillis. A highly Ca2+-sensitive pool of granules is regulated by glucose and protein kinases in insulin-secreting INS-1 cells. J. Gen. Physiol., 124(6):641651, December 2004. 